Hautarzt $2021 \cdot 72: 314-320$ https://doi.org/10.1007/s00105-020-04731-y Online publiziert: 2. Dezember 2020

(c) Der/die Autor(en) 2020

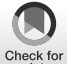

W. G. Philipp-Dormston ${ }^{1,2} \cdot$ R. Aschoff ${ }^{3} \cdot$ T. von Braunmühl ${ }^{4} \cdot$ T. Eigentler $^{5}$.

T. Haalck ${ }^{6} \cdot$ K.-M. Thoms ${ }^{7}$

${ }^{1}$ Fakultät für Gesundheit, Universität Witten/Herdecke, Witten, Deutschland

${ }^{2}$ Hautzentrum Köln, Klinik Links vom Rhein, Köln, Deutschland

${ }^{3}$ Klinik und Poliklinik für Dermatologie, Universitätsklinikum Carl Gustav Carus Dresden, Dresden, Deutschland

${ }^{4}$ Praxis für Dermatologie und Allergologie im Isarklinikum München, München, Deutschland

${ }^{5}$ Zentrum für Dermatologische Onkologie, Universitätsklinikum Tübingen, Tübingen, Deutschland

${ }^{6}$ Fachbereich Dermatologie, Ambulanzzentrum des UKE GmbH - Medizinisches Versorgungszentrum (MVZ) des Universitätsklinikums Hamburg-Eppendorf (UKE), Hamburg, Deutschland

${ }^{7}$ Hautkrebszentrum der UMG/Klinik für Dermatologie, Venerologie und Allergologie, Universitätsmedizin Göttingen (UMG), Göttingen, Deutschland

\title{
Entscheidungskriterien und Patientencharakteristika zur patientenorientierten Therapie der Feldkanzerisierung
}

\section{Ein standardisierter Bewertungsalgorithmus für individuelle Behandlungskonzepte}

\begin{abstract}
Aktinische Keratosen treten häufig chronisch rezidivierend und multilokulär im Sinne einer Feldkanzerisierung auf. Viele der Überlegungen, die wir im Alltag zur Einschätzung des Therapieerfolgs bzw. einer Therapieentscheidung heranziehen, wurden bislang nicht systematisch evaluiert. Um eine gleichermaßen patientenbezogene wie sinnvolle und nachhaltige Therapieentscheidung treffen zu können, bietet sich die Erarbeitung von Patiententypologien und patientenorientierten Entscheidungskriterien an.
\end{abstract}

\section{Hintergrund}

Aktinische Keratosen (AK) gehören zu den häufigsten Hautläsionen im dermatologischen Alltag [1]. In einer Studie aus Deutschland betrug die standardisierte Prävalenz der AK 2,7 \% auf Basis einer großen Kohorte von Arbeitnehmern bzw. 1,8\% auf Basis von 4,5 Mio. Versicherungsdaten. Die Häufigkeit stieg mit zunehmendem Alter an - von 1,6\% im
Alter von bis zu 60 Jahren auf $8,2 \%$ bei den 80- bis 89-Jährigen [2].

AK entstehen in erster Linie durch die kumulative Exposition gegenüber ultravioletter (UV) Strahlung und finden sich daher überwiegend an chronisch lichtexponierten Hautarealen, wie z. B. Stirn, Halsbereich, Arme oder Handrücken [3, 4]. Faktoren wie steigende Lebenserwartung, geändertes Freizeitverhalten, Umweltveränderungen und die damit verbundene wachsende kumulative Sonnenlichtexposition dürften zu einer weiterhin wachsenden Prävalenz der AK beitragen $[5,6]$. Eine hohe Prävalenz von $A K$ lässt sich zudem bei Patienten beobachten, die auf eine chronische Immunsuppression angewiesen sind [7].

AK-Läsionen können in unterschiedlichen Stadien (kategorisiert z. B. klinisch I-III nach Olsen et al. [8] oder histologisch nach Röwert-Huber et al. [9]) auftreten, wobei sie häufig ein ganzes Hautareal (Feldkanzerisierung) betreffen, das neben sichtbaren auch subklinische AKLäsionen umfasst, bei denen die gleichen molekulargenetischen Veränderun- gen vorliegen $[5,10]$. Auch wenn es derzeit nicht möglich ist, das individuelle Progressionsrisiko einer spezifischen AK-Läsion in ein Plattenepithelkarzinom (SCC) vorherzusagen, müssen $\mathrm{AK}$ als wichtiger prädiktiver Marker für die Entwicklung eines nichtmelanozytären malignen Hauttumors (NMSC) bzw. als häufigste Vorstufe für ein SCC gewertet werden $[11,12]$. Als Behandlungsoptionen stehen chirurgische, physikalisch destruierende Maßnahmen oder topische arzneimittelbasierte Verfahren zur Verfügung. Zur Entwicklung eines allgemeingültigen Therapiestandards haben verschiedene Arbeitsgruppen eigene Leitlinien entwickelt $[3,6,13]$. Die aktuelle deutsche S3-Leitlinie „Aktinische Keratose und Plattenepithelkarzinom der Haut “ [14] wurde im März 2020 (Leitlinienreport; Juni 2019) erstellt und hat eine angedachte Gültigkeit von 5 Jahren. Ein von Onkoderm e. V. entwickelter Therapiealgorithmus zur Behandlung von aktinischen Keratosen und insbesondere der Feldkanzerisierung zeichnet sich durch 
Tab. 1 Entscheidungskriterien für patientenindividuelle Therapie

\begin{tabular}{|c|c|}
\hline $\begin{array}{l}\text { Entscheidungs- } \\
\text { kriterium }\end{array}$ & Definition \\
\hline Effektivität & Evidenz zur Wirksamkeit? \\
\hline Selektivität & $\begin{array}{l}\text { Werden subklinische Läsionen mit erfasst? } \\
\text { Bleiben gesunde Zellen weitestgehend intakt? }\end{array}$ \\
\hline Nachhaltigkeit & $\begin{array}{l}\text { Evidenz zu verzögerter oder reduzierter Rekurrenz verfügbar? } \\
\text { Reduktion der Progressionsrate zum SCC? }\end{array}$ \\
\hline Sicherheit & $\begin{array}{l}\text { Gibt es systemische Resorption/Toxizität? } \\
\text { Gibt es Lokalreaktionen? } \\
\text { Ist die Behandlung schmerzhaft? }\end{array}$ \\
\hline Wechselwirkungen & Welche Wechselwirkungen sind zu beachten? \\
\hline Wiederholbarkeit & Wiederholte Anwendung möglich und sinnvoll? \\
\hline Therapiedauer & Dauer der Anwendung? \\
\hline Planbarkeit & $\begin{array}{l}\text { Wie gut lassen sich die Therapiedauer und voraussichtliche Abheilung der } \\
\text { Läsionen mit dem Lebens- und Berufsalltag vereinbaren? }\end{array}$ \\
\hline Kosmetik & Wie ist das kosmetische Ergebnis langfristig? \\
\hline Lebensqualität & Wie wirkt sich die Therapie auf die Lebensqualität aus? \\
\hline
\end{tabular}

eine besonders hohe Praxisrelevanz aus [15].

Angesichts der Vielfalt der verschiedenen Therapieoptionen einerseits und der Heterogenität des Patientenspektrums andererseits setzt die Therapieplanung die Kenntnis therapiespezifischer Vorund Nachteile und ihre individuelle Abwägung voraus. Aspekte wie Lebensqualität, Praktikabilität der Therapie, aber auch Therapieadhärenz, kosmetisches Ergebnis, Patientenpräferenz, Komorbiditäten oder Selektivität einer Therapie sind neben der Effektivität und Sicherheit weitere wichtige Faktoren. Ziel dieser Arbeit war es daher, Kriterien zu erarbeiten, die sich für eine pragmatische Einordnung der zur Verfügung stehenden AKFeldtherapien eignen, und diese anhand dreier definierter AK-Patientenprofile $\mathrm{zu}$ evaluieren, um damit patientenorientierte Therapieentscheidungen treffen zu können.

\section{Methodik}

Die Arbeitsgruppe setzte sich aus Experten auf dem Gebiet der AK-Therapie zusammen $(n=6)$, welche die ambulante Versorgung von NMSC-Patienten an allen Versorgungsstrukturen (Universitätsklinik, medizinisches Versorgungszentrum, niedergelassene Facharztpraxis) länderübergreifend (Dresden, Hamburg, Göttingen, Köln, München, Tü- bingen) abbilden. Alle Teilnehmer des Expertenpanels haben einen dermatoonkologischen Schwerpunkt und verfügen über langjährige und regelmäßige Anwendererfahrungen in der Therapie der Feldkanzerisierung. Die Mitglieder der Arbeitsgruppe wurden gebeten, patientenrelevante Aspekte in der aktuellen Therapielandschaft der AK zu analysieren und auf Basis von erstellten Fallvignetten zu folgenden Fragen Stellung zu beziehen:

1. Nach welchen patientenrelevanten Kriterien sollte die Therapiewahl beim AK-Patienten erfolgen? (Was ist aus Patientensicht wichtig? Was hilft im Therapiealltag?)

2. Welche Patienten sind im Therapiealltag häufig anzutreffen, und bei welchen Patienten ist eine feldgerichtete Therapie sinnvoll? (Welche Patientenmuster weisen ein erhöhtes Risikoprofil für die Feldkanzerisierung auf? Welche AK-Patientenrisikoprofile stellen eine therapeutische Herausforderung dar?)

3. Welches Therapie- und Einsatzprofil ergibt sich aus der beispielhaften Beurteilung einer AK-Therapieoption auf Basis der oben definierten Entscheidungskriterien und Patiententypologien? Als praxisrelevante Beispiele dienten die photodynamische Therapie (PDT) sowie Behandlungen mit Imiquimod, Ingenolmebutat und Diclofenac-Natrium. (Erfüllt die jeweilige Therapie das therapeutische Anforderungsprofil für diese Patienten?)

Die Fragestellungen 1 und 2 wurden beim ersten Arbeitstreffen im Oktober 2017 im Rahmen eines nominalen Gruppenprozesses sowie mehrerer folgender Telefonkonferenzen formuliert. Auf Basis eines Ergebnisprotokolls und der Statements wurden Entscheidungskriterien und Patiententypen in einer ersten Entwurfsversion zusammengefasst und von den Mitgliedern der Arbeitsgruppe kommentiert. Beim zweiten Arbeitstreffen im Oktober 2018 wurde die kommentierte Version im Rahmen eines strukturierten Gruppenprozesses insbesondere hinsichtlich Fragestellung 3 bearbeitet. Ergebnisse und Kommentare wurden 2019 in einem strukturierten Multi-level-Prozess eingearbeitet, 2020 erneut evaluiert und kommentiert, bevor die endgültige Version in der vorliegenden Form konsentiert wurde. Im Rahmen der erweiterten und vertieften Evaluation wurde eine exemplarische Eignungsund Fallbewertung zur Tageslicht-PDT (Daylight-PDT, DL-PDT) durchgeführt. Der Hersteller des Ingenolmebutat-basierten Arzneimittels hat 01/2020 in Abstimmung mit der EMA (Europäische Arzneimittel-Agentur) beschlossen, die Zulassung ruhen zu lassen und das Präparat in Deutschland nicht mehr auszuliefern, weshalb diese ebenfalls untersuchte Behandlungsoption keinen Einzug in die finale Fassung fand.

\section{Ergebnisse}

\section{Entscheidungskriterien für die Therapiewahl}

Die • Tab. 1 führt die gemäß dem Konsens in der Arbeitsgruppe wesentlichen, therapiebezogenen Kriterien auf, die patientenrelevante Fragestellungen umfassen und eine individuelle Beurteilung einer für den Therapiealltag gut geeigneten AK-Therapieoption ermöglichen. Ist der Patient präferenziell auf eine feldgerichtete Therapie der AK angewiesen (bei multiplen AK-Läsionen/ Feldkanzerisierung), sollte die Thera- 
Hautarzt 2021 · 72:314-320 https://doi.org/10.1007/s00105-020-04731-y

(c) Der/die Autor(en) 2020

\section{Entscheidungskriterien und Patientencharakteristika zur patientenorientierten Therapie der Feldkanzerisierung. Ein standardisierter Bewertungsalgorithmus für individuelle Behandlungskonzepte}

\section{Zusammenfassung}

Hintergrund. Aktinische Keratosen (AK) zeichnen sich durch einen chronischen Verlauf aus, und häufig ist ein ganzes Hautareal betroffen (Feldkanzerisierung). Die patientenindividuelle Abwägung therapiespezifischer Vor- und Nachteile einer feldgerichteten Therapie ist herausfordernd.

Fragestellung. Ziel der vorliegenden Arbeit war die Entwicklung und Evaluierung patientenorientierter Entscheidungskriterien, die sich für die pragmatische Einordnung einer AK-Feldtherapie im Behandlungsalltag bei Patienten mit besonderer Prädisposition zur Feldkanzerisierung eignen (Patiententyp 1 bis 3).

Material und Methoden. Die Entwicklung der Entscheidungskriterien und der
Patiententypologie erfolgte im Rahmen eines nominalen bzw. strukturierten Multilevel-Gruppenprozesses. Anhand der patientenrelevanten Entscheidungskriterien, der verfügbaren Evidenz aus klinischen Studien und entlang der Patiententypologie wurde ein Bewertungsalgorithmus etabliert, und feldgerichtete AK-Therapieoptionen wurden systematisch evaluiert.

Ergebnisse. Als patientenrelevante Kriterien für die Therapieentscheidung wurden u.a. Effektivität, Sicherheit, Praktikabilität der Therapie, Adhärenz, Kosmetik, Patientenpräferenz und Komorbiditäten identifiziert und näher spezifiziert. In Bezug auf diese Entscheidungskriterien und Patiententypen, bei denen eine Feldtherapie vorrangig indiziert ist, erfüllte die photodynamische Therapie mit Tageslicht das therapiebezogene Anforderungsprofil in besonderem Maße. Schlussfolgerung. Die Definition von patientenrelevanten und therapiebezogenen Entscheidungskriterien in der AK-Feldtherapie erlaubt eine strukturierte und gleichzeitig praxisorientierte Herangehensweise, um spezifische Therapieoptionen einzuordnen und individuelle Therapieentscheidungen herzuleiten.

Schlüsselwörter

Aktinische Keratosen · Patientenprofile . Lebensqualität · Patiententypologie . Therapieoptionen

\section{Decision criteria and patient characteristics for patient-oriented treatment of field cancerization. Standardized algorithm for personalized treatment concepts}

\section{Abstract}

Background. Actinic keratosis (AK), which frequently affects larger skin areas (field cancerization), is characterized by chronic course. Weighing therapy-specific advantages and disadvantages of field-oriented therapy for individual patients is challenging. Objectives. The main objective was the development of patient-oriented decision criteria for the pragmatic classification of fielddirected AK treatment options in patients with a predisposition for field cancerization (patient types 1-3).
Materials and methods. The development of the decision criteria and patient typology was based on a nominal respectively structured multilevel group process. The developed algorithm was then subsequently applied for the systematic evaluation of field-directed AK therapies.

Results. Patient-relevant criteria for the treatment decision included (among others): effectiveness, selectivity, safety, duration of therapy, cosmesis, patient preference and comorbidities. With regard to the decision criteria and patient types in which field therapy was the treatment of choice, daylight photodynamic therapy notably met the requirement profile.

Conclusion. The definition of patient-relevant and therapy-related decision criteria in AK field therapy allows a systematic yet practice-oriented approach to classify specific treatment options and to derive individual treatment decisions.

Keywords Actinic keratosis - Patient profiles · Quality of life · Patient typology $\cdot$ Treatment options pieoption auch subklinisch betroffene, chronisch UV-geschädigte Hautareale mit erfassen, aber gesunde Zellen weitestgehend intakt lassen (Selektivität). Rezidivpräventive Eigenschaften, Sicherheit (Fokus auf Lokalreaktionen, Schmerzhaftigkeit, systemische Toxizität v.a. bei immunsupprimierten Patienten), Wechselwirkungen, Wiederholbarkeit und Dauer der Therapie oder Planbarkeit sind auch dem chronischen Verlaufscharakter der AK geschuldet, der oftmals wiederholte Therapiezyklen erforderlich macht. Dies impliziert, dass die Behandlung auch bei wiederholter Anwendung für den Patienten sowohl im Hinblick auf unerwünschte Nebenwirkungen, kosmetisches Ergebnis oder Praktikabilität akzeptabel ist und sich nicht ungünstig auf die Lebensqualität auswirken sollte.

\section{Patiententypologien in der ambulanten Versorgung}

Die Teilnehmer der Arbeitsgruppe haben prägnante Patientenprofile erarbeitet und 3 typische Risikopatiententypen (Typ 1 bis 3) mit Prädisposition zur Feldkanzerisierung definiert (•Tab. 2). Bei diesen Patiententypen ist eine feldgerichtete Therapie vorrangig indiziert:

- Typ 1: z.B. der 61-jährige leitende Angestellte, der „schon immer sportlich aktiv" war, umfassend über die Erkrankung, Präventions- und Therapiemöglichkeiten aufgeklärt werden möchte und an „modernen“ Therapieoptionen interessiert ist, die sich zügig umsetzen lassen, sich kosmetisch nicht nachteilig auswirken und 


\section{Typ 1}

Leistungsorientiert, aktiv und gut

informiert

\begin{tabular}{|c|c|}
\hline Geschlecht & Männer und Frauen \\
\hline Bildungsstand & Hoch \\
\hline $\begin{array}{l}\text { Sozioökonomischer } \\
\text { Status }\end{array}$ & Hoch \\
\hline $\begin{array}{l}\text { Kumulative UV-Ex- } \\
\text { position }\end{array}$ & Hoch (+) durch aktives Freizeitverhalten \\
\hline Informationsstand & $\begin{array}{l}\text { Umfassender Aufklärungsbedarf über } \\
\text { Erkrankung und Therapiemöglichkeiten/ } \\
\text { Risiken }\end{array}$ \\
\hline $\begin{array}{l}\text { Kosmetischer An- } \\
\text { spruch an Therapie }\end{array}$ & Hoch \\
\hline $\begin{array}{l}\text { Patientenwünsche/ } \\
\text { Besonderheiten }\end{array}$ & $\begin{array}{l}\text { Kurze Ausfallzeiten, um berufliche/private } \\
\text { Einschränkungen zu minimieren }\end{array}$ \\
\hline
\end{tabular}

Patienten sind dazu angehalten, im Freien auf konsequenten Sonnenschutz zu achten
Typ 2

Typ 3

Aufgeklärter, immunsupprimierter Patient

\begin{tabular}{ll}
\hline Überwiegend Männer & Männer und Frauen \\
\hline Niedrig bis mittel & Alle \\
\hline Niedrig bis mittel & Alle
\end{tabular}

Hoch (+) durch berufliche Tätig- $\quad(+/-)^{\mathrm{a}}$

keit

Tendenziell geringere Krankheits- und Therapieeinsicht

Niedrig bis mittel

Kurze Therapiedauer (ggf. eingeschränkte Therapieadhärenz)
Hoher Wissensstand, Routine im Umgang mit

Niedrig bis hoch

Gespräch auf Augenhöhe, Sicherheit in der AK-Therapie (ohne systemische Nebenwirkungen) Ärzten/Diagnosen; hohe Therapieeinsicht mit einem vollen Terminkalender $\mathrm{zu}$ vereinbaren sind;

- Typ 2: z. B. der 50-jährige subjektiv beschwerdefreie Dachdecker, bei dem im Rahmen des Gesundheitschecks zufällig eine AK diagnostiziert wird, der bezüglich der Therapienotwendigkeit wenig einsichtig ist und bei längerer Therapiedauer voraussichtlich non-adhärent sein wird;

- Typ 3: z. B. der 55-jährige, vor 4 Jahren nierentransplantierte Patient, der bei der regelmäßigen Checkup-Untersuchung mit AK diagnostiziert wurde, gut über das erhöhte Hautkrebsrisiko unter Langzeitimmunsuppression informiert ist und das Für und Wider der AKTherapieoptionen abwägen möchte.

\section{Erfüllt die photodynamische Therapie mit Tageslicht das Anforderungsprofil einer patientengerechten Feldtherapie?}

Als praktisches Anwendungsbeispiel diente die photodynamische Therapie mit Tageslicht (DL-PDT), der in dieser Form jüngste Therapieansatz zur feldgerichteten AK-Therapie. Die Bewertung der DL-PDT auf der Basis der Studienevidenz und entlang der entwickelten Kriterien für eine patientenindividuelle Therapie, wurde systematisch für die jeweiligen definierten Patientenprofile durchgeführt.
Hinsichtlich der Risikopatiententypologie für die Feldkanzerisierung erfüllte die DL-PDT die Kriterien für eine effektive Therapie mit vergleichsweise geringen Rezidivraten [16, 17] und trägt damit zu einer signifikanten Steigerung der Lebensqualität bei [18].

Ein besonderer Vorteil des photodynamischen Wirkmechanismus ist seine hohe Selektivität, welche die Behandlung großer Hautareale erlaubt, die Mitbehandlung subklinischer AK-Läsionen ermöglicht und somit einen hohen Stellenwert in der Prävention von NMSC aufweist [19]. Hiervon profitieren alle Patiententypen, wenn auch der Nutzen gerade für den aufgeklärten, immunsupprimierten Patienten (Typ 3) besonders groß ist.

Die kurze (plausibel vermittelbare) Therapiedauer bzw. Anwenderfreundlichkeit steigert beim Typ 2 die Behandlungsadhärenz, ermöglicht dem beruflich stark eingespannten Typ 1 eine kurze Ausfallzeit und lässt sich ebenfalls in hohem Maße mit der erhöhten Arztbesuchfrequenz des Typ 3 vereinbaren. (• Abb. 1 stellt den zeitlichen Aufwand der zugelassenen Therapieoptionen der Feldkanzerisierung vergleichend dar.)

Da die Belichtung im Vergleich zur konventionellen Rotlicht-PDT aufgrund der ausbleibenden Anreicherung von Protoporphyrin IX weitestgehend schmerzfrei ist, eignet sich die DL-PDT in besonderer Weise für große Hautarea- le, was wiederum die Notwendigkeit für Mehrfachbehandlungen reduziert [20].

Für die Sicherheit spricht, dass wegen fehlender systemischer Resorption diesbezüglich keine Risiken zu erwarten sind $[16,17]$, wovon insbesondere der Typ 3 profitiert, wohingegen das fehlende Narbenrisiko und die hervorragenden kosmetischen Behandlungsergebnisse einen besonderen Mehrwert für den Typ 1 darstellen [21].

Da der Zeitpunkt und die Dauer der lokal auftretenden Hautreaktionen weitestgehend vorhersagbar sind, ist bei der DL-PDT auch die Planbarkeit der Therapie gegeben, was ebenfalls für den Typ 1 von besonderer Bedeutung ist [20].

Allerdings bedarf es vor der ersten Behandlung einer ausführlichen Aufklärung zu Wirkmechanismus und Therapieablauf. Ein entscheidender Nachteil der PDT mit natürlichem Tageslicht sind die jahreszeitlichen und klimatischen Einschränkungen (März bis Oktober, Außentemperaturen $>10^{\circ} \mathrm{C}$, kein Regen). Hier hat sich die artifizielle DLPDT, auch simulierte Indoor-DL-PDT genannt, bewährt, die neben dem Vorteil der Wetterunabhängigkeit auch keinen zusätzlichen Sonnenschutz erfordert, da die gängigen Belichtungseinheiten zwar das Tageslichtspektrum simulieren, allerdings ohne den ultravioletten Anteil [22]. Nicht zuletzt aufgrund der besseren Standardisierbarkeit, der reproduzierbaren gleichen Wellenlängen und 


\section{Originalien}

$$
\begin{array}{lll}
\text { IMQ 3,75\% und 5\% Creme } & (4 \text { Wochen })^{\mathrm{a}} \\
12 & (4-8 \text { Wochen })^{\mathrm{b}}
\end{array}
$$

\section{$(4-8 \text { Wochen })^{b}$}

\section{DFN 3\% Gel}

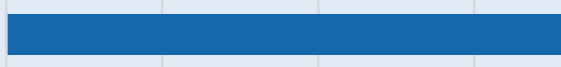

(12,9 Wochen)

5-FU 5\% Creme

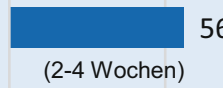

56

(2-4 Wochen)

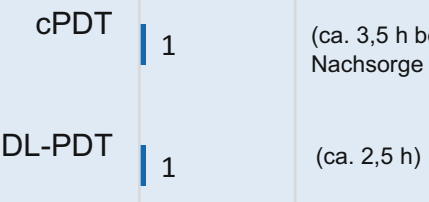

$\begin{array}{llll}0 & 50 & 100 & 150\end{array}$

Abb. $1 \Delta$ Feldgerichtete topische AK(aktinische Keratosen)-Therapien: Applikationsrate (jeweils maximale Anzahl) und Therapiedauer im Vergleich. DFNDiclofenac-Natrium, 5-FU 5-Fluorouracil, IMQ Imiquimod, PDT photodynamische Therapie ( $c_{\text {" }}$ conventional red light", $D L$ Daylight) bei geeigneten Außentemperaturen $\left(>10^{\circ} \mathrm{C}\right.$ ) und Wetterbedingungen (März bis Oktober, wolkenloser bis bedeckter Himmel, kein Regen). 'Zuzüglich Therapiepause von 2 Wochen zwischen ggf. 2 Therapiezy-

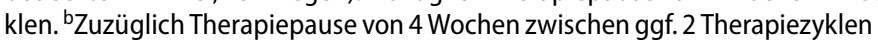

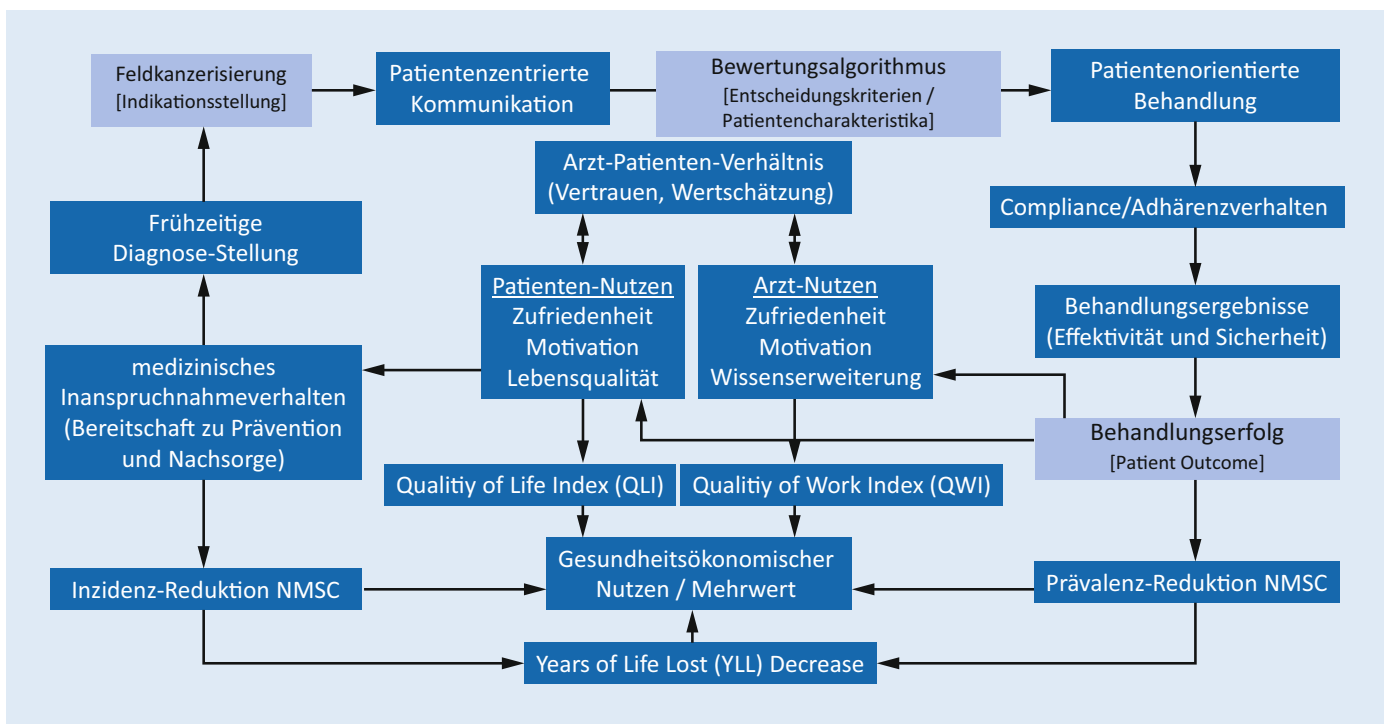

Abb. $2 \varangle$ Integrativer Ansatz der evidenzbasierten und patientenorientierten Therapie der Feldkanzerisierung. NMSC nichtmelanozytärer maligner Hauttumor

der Lichtenergie sowie des Ausbleibens unvorhersehbarer äußerer Umwelteinflüsse ist diese Art der Tageslicht-PDT als besonders sicher und effektiv $\mathrm{zu}$ werten, was erstmals durch die klinischen Daten der Arbeitsgruppe von U. Reinhold mit der ersten standardisierten Indoor-Lichteinheit überhaupt beschrieben werden konnte [23]. Heutzutage stehen verschieden LED-Lichtquellen zur artifiziellen Tageslicht-PDT in Innenräumen zur Verfügung. Eine ak- tuelle Studie aus der Arbeitsgruppe von T. Dirschka hat den hohen Stellenwert dieser Art der Feldtherapie nochmals bestätigt [24]. Das hier angewandte Behandlungsprotokoll wurde mit $1 \mathrm{~h}$ Aminolävulinsäure(ALA)-Inkubation, gefolgt von einer weiteren Stunde Belichtung in einer neuen Tageslichtbehandlungskabine $(20.000 \mathrm{~lx}$, emittierte Wellenlängen an die PP [Protoporphyrin] IXAbsorptions-Peaks angepasst: $457 \mathrm{~nm}$, $523 \mathrm{~nm}, 593 \mathrm{~nm}, 631 \mathrm{~nm}$ ) durchgeführt.
Dieses adaptierte neue Behandlungsprotokoll erwies sich als sicher, beinahe schmerzfrei und sehr effektiv im Hinblick auf die Reduktion des „AK area and severity index" (AKASI) und der Läsionsanzahl [24].

\section{Diskussion}

Zusammenfassend wurde mit der vorliegenden Arbeit ein patienten- und praxisorientierter Algorithmus zur The- 
rapieentscheidung in der AK-Feldtherapie entwickelt. Die Charakterisierung von Patiententypen nach psychosozialen Faktoren kann das Patientenmanagement bei chronischen Erkrankungen erleichtern, indem sie dem Behandler Selektionskriterien aufzeigt, die über die alleinige Erhebung von biomedizinischen Parametern hinausgehen. Wie die Evaluation am Beispiel der DLPDT zeigt, lassen sich therapie- und patientenspezifische Aspekte anhand der Entscheidungskriterien pragmatisch einordnen und bezüglich der vorliegenden Studiendaten diskutieren. Unseres Wissens ist ein vergleichbarer Entscheidungsalgorithmus bisher nicht verfügbar gewesen.

Die Charakterisierung von Patiententypen, die auch psychosoziale Merkmale (z. B. Typ-A- und Typ-D-Persönlichkeit) mit einbezieht, erfährt bei chronischen, kardiovaskulären Erkrankungen nicht nur das zunehmende Interesse der Patienten, Leistungserbringer oder Kostenträger, sondern hat bereits Eingang in Leitlinien und Positionspapiere der Fachgesellschaften gefunden. Der integrative Ansatz kann eine patientenzentrierte Kommunikation fördern, die wiederum positive Effekte auf Patientenzufriedenheit, Adhärenz- und medizinisches Inanspruchnahmeverhalten oder auf Behandlungsergebnisse haben kann [25, 26]. Mögliche Ansatzpunkte, die auch beim dermatologischen AK-Patienten für eine Stratifizierung nach Patiententypologie sprechen würden, ergeben sich aus der epidemiologischen Entwicklung, Chronizität der Erkrankung, Heterogenität des Patientenpools sowie der fehlenden Möglichkeit, zwischen AKLäsionen mit und ohne Rezidiv- oder Progressionsrisiko zu unterscheiden. Trotz der zum Teil umfangreich publizierten Studienliteratur zu einzelnen Therapieverfahren der AK lassen sich solche Ergebnisse nur bedingt miteinander vergleichen, da u. a. verschiedene Endpunkte gewählt wurden bzw. kritische Endpunkte fehlen, die Studien nur selten direkte Vergleiche enthalten oder über keine ausreichende Power verfügen. Die Komplexität eines multimodalen patientenorientierten Ansatzes zum Management der Feldkanzerisie- rung und der mögliche Stellenwert eines Bewertungsalgorithmus im ganzheitlichen Kontext sind in $\bullet$ Abb. 2 dargestellt.

Der systematisch entwickelte und konsentierte Bewertungsalgorithmus soll dabei Entscheidungskriterien aufzeigen, um anhand der existierenden Studienlage das jeweils geeignete Therapieverfahren auszuwählen und hierdurch den Behandlungserfolg bei der Feldkanzerisierung durch eine patientenorientierte Therapie zu steigern.

\section{Fazit für die Praxis}

- Patientenorientierte Entscheidungs-
kriterien können die Einschätzung
des praktischen Stellenwerts einer
Therapieoption bei AK(aktinische
Keratosen)-Patienten erleichtern.
- Die Einordnung einer AK-Thera-
pieoption entlang der Patienten-
typen mit erhöhter Prädisposition
zur Feldkanzerisierung sowie un-
ter Berücksichtigung der aktuellen
Studienlage bietet sich für die Eva-
luierung verschiedener topischer
Therapiemodalitäten an.
Ähnlich wie bei verschiedenen chro-
nisch internistischen Erkrankungen
bereits zu beobachten, lassen sich
Patiententypologien möglicherweise
auch bei der chronischen AK zur
Umsetzung integrativer Behand-
lungskonzepte nutzen.

\section{Korrespondenzadresse}

Dr. med. W. G. Philipp-Dormston

Hautzentrum Köln, Klinik Links vom Rhein Schillingsrotter Str. 39-41, 50996 Köln, Deutschland

mail@haut-zentrum.com

Funding. Open Access funding enabled and organized by Projekt DEAL.

\section{Einhaltung ethischer Richtlinien}

Interessenkonflikt. Die Treffen der Expertengruppe und die Vorbereitung des Manuskriptes wurden durch LEO Pharma unterstützt. W.G. Philipp-Dormston gibt an, Honorare für wissenschaftliche Vorträge, klinische Studien und Beratungstätigkeiten von AbbVie/ Allergan, Almirall-Hermal, Beiersdorf/Eucerin, Benevi, Biofrontera, Galderma, La Roche-Posay, LEO Pharma, Merz und SkinCeuticals erhalten zu haben. R. Aschoff gibt an, Honorare für wissenschaftliche Vorträge und Beratungstätigkeiten von LEO Pharma, Biofrontera, Galderma und Meda erhalten zu haben. T. von Braunmühl gibt an, Honorare von Almirall-Hermal, LEO Pharma und Meda Pharma erhalten zu haben. T. Eigentler gibt an, Honorare für Beratungstätigkeiten von BristolMyers Squibb, Roche, Novartis, LEO Pharma, Pierre Fabre und MSD erhalten zu haben. Darüber hinaus erhielt er Honorare für wissenschaftliche Vorträge von Roche, MSD, Bristol-Myers Squibb und Novartis. K.-M. Thoms gibt an, Honorare für wissenschaftliche Vorträge und Beratungstätigkeiten von LEO Pharma, Galderma, Bristol-Myers Squibb, MSD, Roche, Novartis, Pierre Fabre und AbbVie erhalten zu haben. T. Haalck gibt an, dass kein Interessenkonflikt besteht.

Für diesen Beitrag wurden von den Autoren keine Studien an Menschen oder Tieren durchgeführt. Für die aufgeführten Studien gelten die jeweils dort angegebenen ethischen Richtlinien.

Open Access. Dieser Artikel wird unter der Creative Commons Namensnennung 4.0 International Lizenz veröffentlicht, welche die Nutzung, Vervielfältigung, Bearbeitung, Verbreitung und Wiedergabe in jeglichem Medium und Format erlaubt, sofern Sie den/die ursprünglichen Autor(en) und die Quelle ordnungsgemäß nennen, einen Link zur Creative Commons Lizenz beifügen und angeben, ob Änderungen vorgenommen wurden.

Die in diesem Artikel enthaltenen Bilder und sonstiges Drittmaterial unterliegen ebenfalls der genannten Creative Commons Lizenz, sofern sich aus der Abbildungslegende nichts anderes ergibt. Sofern das betreffende Material nicht unter der genannten Creative Commons Lizenz steht und die betreffende Handlung nicht nach gesetzlichen Vorschriften erlaubt ist, ist für die oben aufgeführten Weiterverwendungen des Materials die Einwilligung des jeweiligen Rechteinhabers einzuholen.

Weitere Details zur Lizenz entnehmen Sie bitte der Lizenzinformation auf http://creativecommons.org/ licenses/by/4.0/deed.de.

\section{Literatur}

1. Rosen T, Lebwohl MG (2013) Prevalence and awareness of actinic keratosis: barriers and opportunities. J Am Acad Dermatol 68:S2-S9

2. Schaefer I, Augustin M, Spehr C et al (2014) Prevalence and risk factors of actinic keratoses in Germany - analysis of multisource data. JEur Acad Dermatol Venereol 28:309-313

3. Werner RN, Stockfleth E, Connolly SM et al (2015) Evidence-and consensus-based (S3) Guidelines for the Treatment of Actinic Keratosis-International League of Dermatological Societies in cooperation with the European Dermatology Forum

4. Kennedy C, Bajdik CD, Willemze R et al (2003) The influence of painful sunburns and lifetime sun exposure on the risk of actinic keratoses, seborrheic warts, melanocytic nevi, atypical nevi, and skin cancer. JInvest Dermatol 120:1087-1093

5. Stockfleth E (2017) The importance of treating the field in actinic keratosis. J Eur Acad Dermatol Venereol 31(Suppl 2):8-11

6. Chetty P, Choi F, Mitchell T (2015) Primary care review of actinic keratosis and its therapeutic options: a global perspective. Dermatol Ther 5:19-35 
7. Stockfleth E, Ulrich C, Meyer T, Christophers E (2002) Epithelial malignancies in organ transplant patients: clinical presentation and new methods of treatment. Recent Results. Cancer Res 160:251-258

8. Olsen EA, Abernethy ML, Kulp-Shorten $C$ et al (1991) J Am Acad Dermatol 24(5 Pt 1):738-743

9. Röwert-Huber J, Patel MJ, Forschner TT et al (2007) Br JDermatol 156(suppl. 3):8-12

10. Braakhuis BJM, Tabor MP, Kummer JA et al (2003) A genetic explanation of slaughter's concept of field cancerization: evidence and clinical implications. Cancer Res 63:1727-1730

11. de Berker D, McGregor JM, Mohd Mustapa MF et al (2017) British Association of Dermatologists' guidelines for the care of patients with actinic keratosis 2017. Br J Dermatol 176:20-43

12. Fernandez Figueras MT (2017) From actinic keratosis to squamous cell carcinoma: pathophysiology revisited. J Eur Acad Dermatol Venereol 31(Suppl 2):5-7

13. Fleming $P$, Zhou S, Bobotsis R, Lynde C (2017) Comparison of the treatment guidelines for actinic keratosis: a critical appraisal and review. J Cutan Med Surg 21:408-417

14. S3-Leitlinie Aktinische Keratose und Plattenepithelkarzinom der Haut, Langversion 1.1 - März 2020, AWMF-Registernummer:032/022OL

15. Onkoderm e.V. Das bundesweite Netzwerk spezialisierter und innovativer Dermatologen. www.onkoderm.de.Zugegriffen:01.12.2020

16. Wiegell SR, Fabricius S, Gniadecka M et al (2012) Daylight-mediated photodynamic therapy of moderate to thick actinic keratoses of the face and scalp: a randomized multicentre study. $\mathrm{Br}$ J Dermatol 166(6):1327-1332

17. Dirschka T, Ekanayake-Bohlig S, Dominicus R et al (2019) A randomized, intraindividual, noninferiority, Phase III study comparing daylight photodynamic therapy with BF-200 ALA gel and MAL cream for the treatment of actinic keratosis. JEur Acad Dermatol Venereol 33(2):288-297

18. Philipp-Dormston WG, Müller $K$, Novak B et al (2018) Patient-reported health outcomes in patients with non-melanoma skin cancer and actinic keratosis: results from a large-scale observational study analysing effects of diagnoses and disease progression. J Eur Acad Dermatol Venereol 32(7):1138-1146

19. Kohl E, Koller M, al Zeman F (2017) Daylight photodynamic therapy versus cryosurgery for the treatment and prophylaxis of actinic keratoses of the face-protocol of a multicenter, prospective, randomized, controlled, two-armed study. BMC Dermatol 17:12

20. Philipp-Dormston WG, Karrer $\mathrm{S}$, Petering $\mathrm{H}$ et al (2015) Daylight PDT with MAL_current data and practical recommendations of an expert panel. JDtsch Dermatol Ges 13:1240-1249

21. Szeimies RM, Lischner S, Philipp-Dormston W et al (2013) Photodynamic therapy for skin rejuvenation: treatment options-results of a consensus conference of an expert group for aesthetic photodynamic therapy.JDtsch Dermatol Ges 11(7):632-636

22. Lerche C, Heerfordt IM, Heydenreich J et al (2016) Alternatives to outdoor daylight illumination for photodynamic therapy - use of greenhouses and artificial light sources. Int J Mol Sci 17(3):309

23. Kellner C, Bauriedl S, Hollstein S et al (2015) Simulated-daylight photodynamic therapy with BF-200 aminolaevulinic acid for actinic keratosis: assessment of the efficacy and tolerability in a re- trospectivestudy. BrJDermatol 172(4):1146-1148. https://doi.org/10.1111/bjd.13420

24. von Dobbeler C, Schmitz L, Dicke Ket al (2019) PDT with PPIX absorption peaks adjusted wavelengths: safety and efficacy of a new irradiation procedure for actinic keratoses on the head. Photodiagnosis Photodyn Ther 27:198-202

25. Albus $C$ et al (2018) K Bedeutung von psychosozialen Faktoren in der Kardiologie - Update 2018. Kardiologe 12:312-331

26. Steca P, Addario M, Magrin ME et al (2016) A type $A$ and type $D$ combined personality typology in essential hypertension and acute coronary syndrome patients: associations with demographic, psychological, clinical, and lifestyle indicators. PLOSONE 11:e161840
Hier steht eine Anzeige. Springer 